\title{
Labyrinthe
}

39 | 2012 (2)

«Et si...? » La cause du contrefactuel

\section{L'usage des raisonnements contrefactuels en histoire}

Pierre Livet

\section{(2) OpenEdition \\ Journals}

Édition électronique

URL : http://journals.openedition.org/labyrinthe/4264

DOI : $10.4000 /$ labyrinthe.4264

ISSN : 1950-6031

Éditeur

Hermann

Édition imprimée

Date de publication : 31 décembre 2012

Pagination : 21-33

ISBN : 9782705682637

\section{Référence électronique}

Pierre Livet, "L'usage des raisonnements contrefactuels en histoire », Labyrinthe [En ligne], 39 | 2012

(2), mis en ligne le 10 janvier 2013, consulté le 19 avril 2019. URL : http://journals.openedition.org/ labyrinthe/4264; DOI : 10.4000/labyrinthe.4264

Propriété intellectuelle 


\title{
L'usage des raisonnements contrefactuels en histoire
}

\author{
Pierre LiveT \\ Université d'Aix-Marseille et CEPERC
}

On a utilisé des raisonnements contrefactuels en histoire dans le but de valider ou de réfuter des relations de causalité. L'exemple le plus connu est celui des travaux de Fogel, sur la question de l'importance du développement des chemins de fer dans l'essor de l'économie américaine. En imaginant des scénarios contrefactuels dans lesquels ce développement n'aurait pas eu lieu, il est arrivé à une conclusion négative : l'essor américain aurait pu avoir lieu même en utilisant d'autres moyens de transport - en l'occurrence les transports fluviaux.

D'autres auteurs se sont demandé si ce qu'une certaine vulgarisation de l'histoire présente comme des événements décisifs - des turning points - mérite bien cette qualification : sans ces événements, le cours des choses aurait-il été changé? On pense au nez de Cléopâtre invoqué par Pascal, même si, malheureusement, ce nez n'était pas, d'après les monnaies frappées à son effigie, l'atout de séduction le plus décisif de Cléopâtre.

Disons tout de suite qu'à notre sens, ces raisonnements sur des scénarios contrefactuels ne peuvent pas espérer répondre positivement à la question : tel événement historique était-il bien une des causes nécessaires d'un autre événement historique ? Pour ce faire, il faudrait disposer d'une théorie des relations causales entre tous les événements, théorie qui puisse nous assurer que la reconstruction contrefactuelle proposée comporte tous les événements pertinents pour donner un contenu aux relations causales indispensables à l'étude du cas considéré. Or, si nous disposions de cette théorie, nous saurions déjà ce qui est cause nécessaire et ce qui ne l'est pas.

On ne peut pas non plus sérieusement espérer montrer que tel événement réduit à sa description singulière (César franchit le Rubicon) est une cause nécessaire des changements de régime qui font passer d'un 
état historique global à un autre, de la République à l'Empire romain. Cet événement singulier a lui-même de multiples causes, et il est fort probable que certaines de ces causes ont une influence importante pour ce qui concerne le changement global de régime, mais qu'elles ne sont pas déterminantes pour la production de cet événement dans sa singularité, alors que celles qui spécifient cette singularité ne sont pas décisives pour le changement global de régime en question. Or, pour savoir quelles sont les causes nécessaires, il faut déjà avoir fait une différence entre les deux types de cause, ce qui là encore revient à supposer le problème résolu.

Il faut, pensons-nous, adopter une position plus prudente. Les raisonnements sur les scénarios contrefactuels nous permettent de comparer plusieurs manières d'ordonner d'hypothétiques causes selon l'importance de leur influence. Ils ne permettent pas de dire que telle hiérarchie de causes est la bonne, mais ils permettent d'avoir des soupçons fondés sur la fragilité de telle hiérarchie relativement à une autre. Au lieu de nous permettre des conclusions absolues et positives, ils nous permettent des conclusions qui sont comparatives et négatives.

Un autre renversement de perspective est alors possible. Il est illusoire de se focaliser sur un événement singulier et de prétendre démontrer que sans lui des changements bien plus globaux n'auraient pas eu lieu. En revanche, il est possible de partir d'un cas singulier, et de déployer à partir de lui le réseau des interactions et des influences qu'il révèle, ce que fait la micro-histoire. On peut alors utiliser des raisonnements contrefactuels pour mieux spécifier la nature de ces interactions et de ces influences, en comprenant ce qu'elles ont été par contraste avec ce qu'elles auraient raisonnablement pu être - si ces scénarios contrefactuels sont au moins partiellement documentés par un autre cas. En spécifiant ces interactions et ces influences, on aura des résultats de portée générale, puisque la plupart des relations de ce réseau ne sont pas seulement propres au cas en question, mais nous informent sur des propriétés relationnelles plus générales de la société du lieu et de l'époque'.

1. Des discussions nous ont amené à partager les positions de Jacques Revel (pour son intérêt, tempéré par sa prudence, pour les développements de l'histoire contrefactuelle) et de Giovanni Levi (pour son épistémologie de la micro-histoire). Ce texte a aussi bénéficié de discussions avec Alain Trannoy. 


\section{L'usage des raisonnements contrefactuels en histoire}

\section{Le raisonnement contrefactuel en histoire et ses critiques}

Pour appuyer ces positions, revenons sur les particularités du raisonnement contrefactuel. Nous nous en tiendrons à sa version logique la plus connue, celle de Lewis-Stalnaker ${ }^{2}$. Un conditionnel contrefactuel est du type «Si A était le cas, alors B serait le cas», alors que l'on sait que A n'est pas le cas. On évalue la vérité de ce conditionnel en considérant un ensemble de mondes possibles, ordonnés selon une relation de proximité ou de similarité par rapport à un monde de référence (le monde actuel, en principe). On se place dans les mondes possibles qui vérifient l'antécédent $\mathrm{A}$, en se restreignant aux mondes possibles les plus proches du monde de référence (il se peut que ces A-mondes ne soient qu'une partie de ces mondes les plus proches), et on vérifie que, dans tous ces A-mondes les plus proches, le conséquent du conditionnel est bien le cas. Le conditionnel est alors validé. Cela revient à dire que si l'on part d'un quelconque de ces A-mondes les plus proches, et que l'on considère tous les mondes accessibles dans cette zone restreinte de mondes, le conséquent y est vrai, autrement dit, que la relation de conséquence mise en place par le conditionnel est nécessaire, d'une nécessité restreinte à cette région et à la notion de similarité ou de proximité utilisée.

Pour raisonner en termes de causalité, on considère le conditionnel «Si C n'était pas le cas, alors E ne serait pas le cas», C étant ce qu'on propose par hypothèse comme cause, et $\mathrm{E}$ étant l'effet observé. La conclusion que l'on souhaite obtenir est que, dans tous les mondes les plus proches où nonC était le cas, mondes ordonnés en référence à cette différence entre $\mathrm{C}$ et nonC, et aux relations qui prévalent dans le monde actuel, nonE est le cas.

Prenons pour exemple la démarche de l'ouvrage de Fogel Railsroad and American Economic Growth (1964). Fogel, on l'a dit, soutient que si le chemin de fer n'avait pas existé, les capitaux se seraient reportés sur les transports fluviaux et maritimes, et que finalement la croissance américaine n'aurait pas été fortement touchée. Il considère donc un monde où les relations économiques seraient les mêmes que dans notre monde, à ceci près que le chemin de fer n'aurait pas été inventé. On aurait cependant pu utiliser des capitaux pour construire des canaux et pour multiplier les transports fluviaux et maritimes, et les calculs de

2. Exposée par exemple dans David K. Lewis, Counterfactuals, Oxford, Blackwell, 1973. 
Fogel montrent alors que le développement économique aurait été un peu ralenti, mais pas arrêté. Le chemin de fer ne serait donc pas une cause nécessaire de ce développement.

Cet ouvrage a donné lieu à bien des controverses. Nous retiendrons ici seulement l'article de Paul David, «Transport Innovation and Economic Growth : Professor Fogel on and off the Rails ${ }^{3} »$, parce qu'il nous permettra, en mettant une de ses objections en relation avec un récent article de Kit Fine sur la sémantique des contrefactuels, de soulever un problème propre aux disciplines qui étudient des événements dépendant de choix humains, parmi lesquels il faut évidemment compter l'histoire.

David note que Fogel n'a pas tenu compte du fait que le rendement des investissements dans les chemins de fer était beaucoup plus élevé qu'il ne l'était dans les transports fluviaux et par canaux, si bien que dans une économie basée sur les transports fluviaux ou par canaux, ce rendement moindre aurait ralenti la croissance plus fortement que ne le soutenait Fogel.

Cela soulève la question de l'influence sur les décisions humaines des comparaisons que les hommes peuvent faire à partir de l'éventail de choix qu'on leur offre. La psychologie expérimentale (dans les travaux de Kahnemann et Tversky, par exemple) nous a montré que nos décisions dépendaient de l'ensemble d'options que l'on nous proposait. Un physicien pourrait, du haut de sa théorie, considérer l'ensemble des options possibles, et non pas seulement celles considérées par, mettons, un automate qui prendrait une «décision». Mais un historien ne peut pas se permettre d'utiliser son savoir des événements subséquents pour reconstruire un éventail de conséquences possibles des différents choix, et prétendre expliquer le cours qu'a pris l'histoire en fonction de cet éventail de conséquences, alors qu'elles ne pouvaient être distinguées par les acteurs de l'époque. Du moins, il ne peut pas le faire quand il s'intéresse aux causes et raisons des choix des acteurs.

En revanche, il semble qu'il puisse et même doive le faire quand il s'intéresse aux déterminants effectifs des conséquences des décisions des acteurs. Il se peut que les acteurs n'aient pas prêté attention au fait qu'ils avaient dépassé le seuil à partir duquel leur exploitation des forêts, ou du gibier ou des possibilités de pêche locales, empêchait la reconstitution des ressources (cas des habitants des Galapagos, des Anasazis, etc.).

3. Economic History Review, 3, 22, 1969. 


\section{L'usage des raisonnements contrefactuels en histoire}

Pour comprendre les causalités qui ont mené au dépérissement ou à la disparition de ces populations, l'historien doit aussi intégrer ces causes.

Le problème est alors de savoir comment combiner des causalités qui n'impliquent que des causes et des effets, qui fonctionnent soit de manière déterministe, soit de manière aléatoire, ce que nous appellerons des «naturalités», et des causalités qui impliquent des choix, lesquels se font en fonction des comparaisons disponibles pour les acteurs.

La critique de David concernant Fogel peut s'interpréter dans le second cadre : les investisseurs historiques ont été attirés par les rendements élevés d'investissements dans les chemins de fer, ce qui a eu un effet accélérateur de la croissance - d'abord pour la construction des chemins de fer, ensuite pour les effets sur les transports de masse. Si ces investisseurs n'avaient pas disposé parmi leurs choix possibles de ces investissements comparativement si attractifs, ils auraient été moins motivés pour investir dans des systèmes de transport moins rentables, et il n'y aurait pas eu cet effet accélérateur, donc la croissance américaine n’aurait pas décollé.

Donnons un exemple de critique qui se situe plutôt dans le premier cadre : Elster a fait en 1975 la critique suivante : si on n'avait pas inventé les locomotives, les régularités observées dans les innovations - dont les unes favorisent les autres - nous permettent de juger que probablement on n'aurait pas non plus inventé le moteur à explosion. La croissance américaine aurait donc été fortement freinée.

Depuis 1975, cependant, et par exemple dans Nuts and Bolts for Social Sciences, Elster a montré ses doutes sur le fait qu'on puisse appliquer des sortes de déterminismes ou de fortes probabilités à ces relations propres au domaine de l'innovation. Les deux avenirs, celui dans lequel on n'aurait pas inventé le moteur à explosion, celui dans lequel on l'aurait inventé, semblent possibles. Mais ce qui provoque une bifurcation dans un sens ou dans un autre n'est pas défini : il existe aussi bien, selon l'expression d'Elster, des petits mécanismes pour nous lancer sur une voie que des petits mécanismes pour nous lancer sur l'autre.

On peut aller plus loin : l'histoire nous montre bien des exemples où la survenue de certaines contraintes nouvelles, qui diminuent les possibilités dont profitaient auparavant les humains, a suscité des activités innovatrices qui ont permis les développements humains. On peut invoquer comme exemples la diminution des pluies en Afrique qui a favorisé la savane, ce qui aurait privilégié la station debout et des possibilités - et 
nécessités - plus grandes de déplacement; des conditions moins favorables qui suscitent des migrations; la construction de canaux d'irrigation sous des contraintes de sécheresse; celle de digues qui ont permis des polders (Pays-Bas); les villes sur pilotis (Venise) qui se tournent vers le commerce maritime, etc. Il y a là des évolutions où, à des contraintes qui restreignent les possibilités, les humains répondent par des actions qui ont pour conséquence l'élargissement des possibles. Cela est difficile à intégrer dans le cadre des «naturalités».

Cependant, pour pouvoir parler de causalité en histoire, il faut pouvoir relier les deux perspectives, celle des naturalités, qui combine processus déterministes et processus aléatoires, et une autre perspective qu'on pourrait dire «focalisatrice», liée aux décisions, qui tient compte des sélections et orientations, donc des focalisations que les acteurs pouvaient activer dans un contexte donné en fonction de leurs raisons et motivations. Ces raisons peuvent être très variées, allant d'une rationalité instrumentale à des considérations religieuses et magiques. Quelles qu'elles soient, elles amènent les acteurs à négliger certains facteurs, à considérer certains facteurs par comparaison avec d'autres, et parfois à se focaliser sur la possibilité de nouveaux facteurs.

Nous avançons l'hypothèse que les historiens sont surtout intéressés par les moments où se greffent sur les causalités «naturelles » des activités focalisatrices (dans nos exemples, les irrigations, les polders, la ville sur pilotis), soit où, au contraire, les activités focalisées voient leurs effets détournés par des naturalités (par exemple : le non renouvellement de la ressource).

\section{Différents régimes de contrefactuels}

Comment formuler ces greffes et ces détournements dans les termes des raisonnements contrefactuels?

C'est ici qu'on peut partir d'une idée avancée par Kit Fine 4 . Il n'est pas satisfait par l'analyse des contrefactuels en termes de mondes possibles. La raison qu'il en donne est que, puisque la sémantique des mondes possibles exige qu'on puisse donner un sens à la vérité (ou fausseté) d'une proposition à travers tous les mondes possibles, on doit admettre

4. A difficulty for the analysis of possible worlds analysis of counterfactuals, in Synthese, March 2012. 
que l'antécédent d'un contrefactuel n'est défini qu'à ses substitutions logiques près. Ainsi, on peut substituer au contrefactuel p > nonq (si p, alors nonq) le contrefactuel :

$(\mathrm{p} \& \mathrm{q})$ ou $(\mathrm{p} \&$ nonq) $>$ nonq.

De là, partant de l'hypothèse d'une chaîne infinie d'éléments (du type dominos), tels que si l'un tombait le suivant tomberait, du contrefactuel qui nous dit que si un domino tombait, le précédent ne tomberait pas, et de l'hypothèse qu'il est contrefactuellement possible qu'un domino tombe, Fine arrive à déduire que si un des dominos tombait, alors ce ne serait pas le premier, mais peut-être le second à partir de lui, et par itération du raisonnement, que ce ne serait aucun des dominos qui tomberait, d'où contradiction.

Le paradoxe lui-même n'est pas directement pertinent pour notre propos (si le physicien peut à la limite se soucier de chaînes de causalité qui n'arrivent pas à trouver un premier terme - il n'a généralement pas à se soucier de celles qui ne lui trouvent pas un dernier terme -, l'historien, lui, ne se soucie pas de chaînes infinies) mais le remède que propose Fine est très suggestif.

Il propose de distinguer entre les conditions générales de vérité d'une assertion, et les éléments qui dans notre monde rendent vraie cette assertion. Par exemple, l'assertion «il y a du vent ou il n'y a pas de vent» et l'assertion «il pleut ou il ne pleut pas », étant des tautologies, ont les mêmes conditions générales de vérité. En termes de mondes possibles, elles sont vraies dans les mêmes mondes possibles. Mais pour savoir ce qui rend vraie la première, il faut chercher des états du monde où il y a du vent, et des états où il n'y a pas de vent, et pour savoir ce qui rend vraie la seconde, il faut chercher des états du monde où il pleut et d'autres où il ne pleut pas, et ce ne sont pas les mêmes états que précédemment, alors que ce sont toujours les mêmes mondes (les mondes où pleuvoir ou ne pas pleuvoir, venter ou ne pas venter sont admis). Si je dispose de ce qui rend vraie une assertion, dit Fine, je dispose d'un vérificateur exact de cette assertion, et si je ne dispose que des conditions générales de vérité, j'ai un vérificateur inexact («imprécis» ou «plus vague» serait plus proche de ce qu'on veut signifier ici).

Il faut donc, nous dit Fine, exprimer l'antécédent et le conséquent du conditionnel contrefactuel dans les termes de tels états. Le problème alors, c'est que nous semblons perdre le rapport entre la liaison conditionnelle et la nécessité de la dépendance qu'elle implique. 
Comment retrouver entre des états du monde une forme de nécessité? En imposant à la liaison entre états du monde qu'on envisage des contraintes maximales, qui, en un sens, miment les relations entre mondes possibles associées à la nécessité. Dans la sémantique des mondes possibles, est nécessaire une proposition vraie dans tous les mondes possibles accessibles à partir du monde de référence. Cette définition prend un monde comme point de départ, et envisage tous les mondes possibles accessibles comme lieux d'arrivée.

Fine va faire de même avec ses états : l'antécédent est d'abord défini sans qu'on se soucie de savoir quel état le rend vrai. Il suffit que ses conditions de vérité, quelles qu'elles soient - ce sont donc des conditions générales de vérité -, soient satisfaites. Mais pour déclencher à partir de cet antécédent la relation de conséquence, on est bien plus exigeant : pour toute manière de rendre vrai l'antécédent (ce que Fine appelle une définition exacte de l'antécédent), la relation de conséquence exprimée par le conditionnel contrefactuel doit trouver une manière de rendre vrai le conséquent. Autrement dit, dans une discussion argumentée, le proposant, celui qui soutiendrait le conditionnel contrefactuel et sa relation de conséquence, n'a pas le choix de spécifier des conditions particulières de vérité pour l'antécédent. En revanche celui qui s'opposerait à son assertion (qui peut être un opposant raisonneur ou tout simplement la Nature) choisit la manière qu'il veut de rendre vrai le conséquent, pour ensuite mettre le proposant au défi de la trouver à partir de l'antécédent. Puisque l'opposant a tout le choix qu'il veut, cela veut dire que de son côté le conséquent n'est déterminé que de manière inexacte. En revanche, ce qu'il exige du proposant, c'est une manière de rendre vraie le conséquent à partir d'une manière de rendre vrai l'antécédent, donc en partant d'une version exacte de l'antécédent. Et le proposant, lui, n'a pas le choix concernant cette manière de rendre vrai l'antécédent. Bref, pour le proposant, ne pas avoir de choix en antécédent, être préparé à tout accepter en conséquent : ce sont là des contraintes maximales.

Il est assez clair qu'il s'agit bien là de la modalité qui correspond à des contraintes maximales : celle de la nécessité, par exemple la nécessité de lois physiques déterministes. Mais nous avons vu qu'il existe un autre mode de naturalité, celui de l'aléatoire : le proposant a alors un certain choix - comme un joueur qui choisit un numéro à la loterie - mais c'est l'opposant (la loterie ou la Nature) qui définit quel conséquent lui est assigné. La contrainte concernant l'antécédent n'est pas maximale, mais 
celle sur le conséquent le reste. Il existe d'autres modalités, celles liées à des choix plus efficients. On peut les définir en combinant différemment contraintes sur l'antécédent et contraintes sur le conséquent. Une autre combinaison, par exemple, serait que le proposant pourrait s'imposer des contraintes fortes (sur l'antécédent) alors que l'opposant ne pourra pas imposer de contraintes maximales sur le conséquent. Dans une autre combinaison, les contraintes seront faibles et sur l'antécédent et sur le conséquent. Distinguons ces modalités en partant de différentes versions d'une situation souvent prise pour exemple par les théoriciens des conditionnels : «si je n'avais pas frotté l'allumette, elle ne se serait pas enflammée » (qui est liée à une naturalité proche de la nécessité).

Naturalité de l'aléatoire. Antécédent : «Si j'avais choisi une autre allumette»; Conséquent : «alors elle se serait probablement allumée ».

On a en antécédent un choix ad libitum - on peut prendre n'importe quelle allumette. Mais c'est l'ensemble probabiliste des dispositions des différentes allumettes à s'allumer qui décide en conséquent : nous devons accepter n'importe quel état de la Nature lié à notre conséquence. «Alea jacta est» disait César (mais en grec, paraît-il).

La nécessité et l'aléa sont les deux régimes d'une relation de conséquence «naturelle», où le proposant n'a pas le choix du conséquent.

Focalisation par auto-imposition de contraintes : «Si tu avais frotté l'allumette, que tu l'aies protégée contre le vent, que tu aies eu de l'herbe sèche, etc. tu aurais allumé le feu » (celui qui parle donne un conseil à un débutant en allumage de feu dans un campement en plein air).

Le conseil est de mettre en acte les restrictions indispensables pour que l'allumette s'allume (pas pour un autre but). On ajoute donc des contraintes en antécédent. En revanche on se focalise seulement sur l'effet visé, celui qui donne une sanction positive ou négative de l'efficacité des conseils qui visent cet effet et qui ne se soucient pas d'autres conséquences. S'il y a d'autres conditions non explicitées, la conséquence en sera tout simplement supposée non pertinente. On procède par focalisation à une auto-limitation de la sphère de pertinence des conseils, qui permet de sélectionner les conséquences tests (en négligeant celles qui ne le sont pas).

Comme exemple historique, plaçons la phrase suivante dans la bouche de Pompée, qui se reprocherait de ne pas avoir suivi le conseil qu'il aurait pu se donner à lui-même s'il avait raisonné comme César. «Si j'avais agi comme César, qui a conservé ses légions en sortant de sa province, 
a soigné leur paie et leur approvisionnement, sans se soucier de l'infraction aux règles politiques romaines, alors j'aurais pu l'emporter sur lui ».

Focalisation à partir d'un éventail de possibilités : «Si tu avais eu du gaz, et des allumettes ou un allume-gaz, tu aurais allumé le réchaud».

Il s'agit d'un choix ad libitum (mais il faut du gaz et l'un ou l'autre des deux autres ingrédients) et il y a focalisation sur un effet (but).

Exemple en histoire : si César Borgia avait pu persuader, ou soudoyer, ou éliminer ses adversaires, il aurait pu obtenir le pouvoir (Machiavel). Nous parlerons de choix contingent.

On voit que les contrefactuels permettent, à côté des modalités du nécessaire et de l'aléatoire, d'exprimer deux autres modalités, qui combinent choix et focalisation, permettant de sélectionner parmi les conséquences celles qui sont pertinentes et de se focaliser dessus.

Revenons alors, munis de ces distinctions entre régimes de causalité, ou plus exactement de conditionnalité contrefactuelle, à la controverse portant sur les thèses de Fogel. Le raisonnement contrefactuel qu'il adoptait présupposait le cadre des naturalités (déterminisme ou bien aléa probabiliste). On peut alors reformuler l'argument de David : si le ressort de la focalisation des investisseurs est un différentiel important dans la comparaison entre les rendements des investissements, allant de pair avec un espoir en un effet d'accélérateur, alors le montant total des investissements quand on ne dispose pas de ces perspectives de fort rendement à plus long terme est moins élevé que quand on en dispose. Ce qui sous-tend ce raisonnement, c'est le passage d'un régime de type 4 (choix ad libitum et focalisation sur le rendement supérieur) à un régime de type 2 (aléa : le joueur choisit son numéro, mais c'est la Nature - la loterie - qui décide).

Le raisonnement initial d'Elster, lui, nous ramenait à des régularités qu'on peut à la limite ranger dans le régime $\mathrm{n}^{\circ} 1$ de contrefactuel, celui d'une forme de nécessité. Notre objection était qu'on a observé dans l'histoire bien des situations où une augmentation de contrainte, au lieu de nous maintenir dans le régime 1 , a conduit au régime 3 , celui où de la contrainte on a pu tirer de l'innovation - les polders, ou encore Venise et sa construction sur un terrain peu stable.

Bien entendu il est toujours possible de revenir de là au régime 2, celui des aléas : il y a à côté du destin de Venise d'autres innovations qui n'ont pas réussi, et cela est lié à une sorte de loterie, qui a fait que seules les innovations réussies nous sont parvenues. On pourrait aussi 
prétendre revenir à un régime de type $1:$ les innovations sont soumises à des contraintes, et les efforts pour satisfaire ces contraintes étant importants, un jour ou l'autre ces efforts dépassent les capacités des collectifs, si bien qu'on assiste à une dégradation des positions obtenues par ces innovations.

\section{Propositions pour un bon usage des contrefactuels en histoire}

Si nous revenons à l'usage que peut faire l'historien des raisonnements contrefactuels, on voit aisément qu'il ne peut se satisfaire des seuls régimes des «naturalités », puisqu'il a affaire à des humains, qui sont capables de choix. Les humains peuvent soit exercer leur « libre arbitre» en choisissant selon leur fantaisie entre différentes possibilités qui leur sont offertes (contraintes minimales sur l'antécédent et sur le conséquent), soit choisir de s'imposer certaines contraintes en antécédent pour pouvoir dans le conséquent transformer le monde de manière plus favorable à leurs buts.

Il est clair que l'historien doit s'intéresser aux rapports entre ces différents régimes. D'une part, il ne peut pas tenir compte uniquement des choix des acteurs (ce que faisait au fond l'histoire «événementielle»), il doit tenir compte des régularités - des ersatz de déterminismes - et des aléas. Mais on ne peut pas tout réduire à des naturalités. S'il est toujours possible en principe d'espérer re-décrire des choix dans les termes de régularités ou d'aléas, il ne faut pas oublier qu'à l'inverse, une re-description des régularités et des distributions probabilistes dans les termes des choix est possible : ce que les scientifiques nous ont fait connaître des naturalités, ils l'ont obtenu par focalisation, puisque la méthode expérimentale consiste à laisser de côté les effets que le scientifique ne considère pas comme des tests pour son hypothèse!

Que peut alors faire l'historien des raisonnements contrefactuels? En fait, selon nous, l'historien ne s'intéresse pas aux causes, ni même simplement aux relations de causalité. Son objet, ce sont ces changements ou articulations entre les différents régimes de causalité que nous avons mis en évidence. Comment peut-il les repérer ou les identifier? Une méthode possible pourrait être de commencer par tenter d'expliquer des relations de conséquence selon le régime de nécessité. Si cela n'est pas convaincant, alors on tenterait de recourir au régime de l'aléa. II resterait des cas comme celui de Venise où la probabilité de réussite restait très 
faible, et où les choses s'expliquent mieux si l'on admet que l'ensemble des conséquences possibles a été modifié par des innovations - par exemple, pour la réussite de Venise, après l'usage des pilotis, la construction dans l'arsenal de galères sur le même type, en utilisant des éléments fabriqués sur le même modèle. Il est encore d'autres situations (par exemple l'afflux d'or en Espagne, qui permettait de choisir ad libitum son utilisation) qui n'incitaient pas à s'imposer les contraintes du régime d'auto-limitation.

Il serait possible ensuite de construire des modèles de chacun de ces régimes de conditionnel. Certes, chacun de ces modèles se présenterait comme une (peudo-) naturalité, puisqu'un modèle détermine de manière forcément restrictive quels choix sont autorisés et quelles relations sont valides entre choix et conséquences. Les modèles ne peuvent pas rendre compte de la créativité des choix humains, si bien qu'ils n'expliquent pas les changements qui lui sont liés. Ces modèles auraient donc une pertinence locale, limitée à une étape de l'évolution historique (par exemple, le modèle de Venise à ses débuts n'est pas le même que celui qui concerne les innovations de l'arsenal). À chaque étape, les contextes à mobiliser peuvent changer : ainsi, pour son développement, Venise a déboisé toutes les forêts de chênes sur son Piémont, si bien que son contexte environnemental a changé. Une fois que Venise s'est lancée dans le commerce international, le fait qu'elle soit le débouché d'une voie de transit qui passe par l'Autriche était évidemment décisif, alors que ce contexte n'était pas pertinent pour un village de pêcheurs, etc.

On pourrait vouloir se poser la question : quelle est la cause qui fait basculer d'un régime de conditionnalité contrefactuelle à un autre? Mais comme nous ne serions plus alors au niveau des causes, mais au niveau des transitions entre régimes de causalité, trouver la «cause» de ces changements de régime serait une question dénuée de pertinence scientifique.

Ces considérations pourraient sans doute aussi avoir quelque pertinence pour la micro-histoire et l'étude de cas. Si nous revenons à la conception des contrefactuels de Lewis et Stalnaker, nous nous souvenons qu'elle présupposait la définition d'une mesure de proximité ou de similarité entre les différents mondes contrefactuels et le monde actuel. Mais on ne savait pas trop sur quoi reposait cette mesure qui définissait l'ordonnancement hiérarchique des mondes possibles. Elle pouvait aussi bien être liée à une priorité donnée à la conservation dans les mondes contrefactuels des lois physiques du monde connu, qu'à des préférences et aux priorités de celui qui tient ces raisonnements contrefactuels, données 


\section{L'usage des raisonnements contrefactuels en histoire}

à tel type de monde plutôt qu'à tel autre. Or la typologie des régimes de contrefactuel que nous avons esquissée nous permet non seulement de différencier ces hiérarchies, mais de relier les différences entre ces hiérarchies à l'imposition ou non de contraintes exigeant une définition «exacte» de l'antécédent ou du conséquent. Cela permet de ne pas laisser la définition de ces priorités dans un vague complet, et d'envisager des règles de passage d'une modalité de hiérarchisation à une autre.

Ainsi l'historien qui part d'une étude de cas qui semble a priori limitée peut cependant espérer, en ayant pris comme hypothèse de départ un régime de raisonnement contrefactuel - par exemple le moins contraignant, celui des choix ad libitum - montrer en quoi certaines contraintes s'imposent à son ou ses acteurs - contraintes qui restreignent l'éventail des choix possibles en antécédent, restrictions dont les acteurs ont conscience. Il peut ensuite discerner parmi ces contraintes conscientes celles que les acteurs reconnaissent comme valides et dont on peut vérifier s'ils se les sont imposées à eux-mêmes, et celles qu'ils attribuent à de bonnes ou mauvaises fortunes. Il reste enfin des contraintes qui ne sont pas forcément mentionnées comme telles dans les documents, mais que de son point de vue d'observateur extérieur l'historien peut connaître, alors même que les acteurs de l'époque n'en ont pas eu conscience. L'étude approfondie et comparée de quelques cas peut ainsi suffire à disposer d'informations décisives pour bien décrire l'articulation des différents régimes, articulations dont la caractérisation a bien une portée générale.

L'historien n'utilise donc pas les raisonnements contrefactuels pour établir positivement et de manière absolue une causalité. Il procède d'abord comparativement et passe d'un régime de contrefactuel à un autre pour des raisons négatives : les modèles qui s'inspirent d'un seul régime n'expliquent pas un certain nombre de données. C'est donc seulement dans une enquête qui détecte ces changements entre régimes de conditionnalité, et qui repose donc sur des distinctions entre différents régimes de contrefactuel, en suivant la piste ouverte par Kit Fine, que les raisonnements contrefactuels peuvent être indispensables au travail de l'historien. 